University of New Hampshire

University of New Hampshire Scholars' Repository

Space Science Center

Institute for the Study of Earth, Oceans, and

Space (EOS)

9-26-2013

\title{
Plans for the next GRAPE balloon flight
}

\author{
Mark L. McConnell \\ University of New Hampshire - Main Campus, mark.mcconnell@unh.edu \\ Peter F. Bloser \\ University of New Hampshire, Peter.Bloser@unh.edu \\ T P. Connor \\ University of New Hampshire - Main Campus \\ Camden Ertley \\ University of New Hampshire - Main Campus \\ Jason S. Legere \\ University of New Hampshire, jslegere@unh.edu
}

See next page for additional authors

Follow this and additional works at: https://scholars.unh.edu/ssc

Part of the Astrophysics and Astronomy Commons

\section{Recommended Citation}

Citation Mark L. McConnell ; Peter F. Bloser ; Taylor P. Connor ; Camden Ertley ; Jason Legere ; James M. Ryan and Sambid K. Wasti " Plans for the next GRAPE balloon flight ", Proc. SPIE 8859, UV, X-Ray, and Gamma-Ray Space Instrumentation for Astronomy XVIII, 885909 (September 26, 2013); doi:10.1117/ 12.2024145; http://dx.doi.org/10.1117/12.2024145

This Conference Proceeding is brought to you for free and open access by the Institute for the Study of Earth, Oceans, and Space (EOS) at University of New Hampshire Scholars' Repository. It has been accepted for inclusion in Space Science Center by an authorized administrator of University of New Hampshire Scholars' Repository. For more information, please contact Scholarly.Communication@unh.edu. 


\section{Authors}

Mark L. McConnell, Peter F. Bloser, T P. Connor, Camden Ertley, Jason S. Legere, James M. Ryan, and Sambid K. Wasti 


\title{
Plans for the next GRAPE balloon flight \\ Mark L. McConnell ${ }^{1}$, Peter F. Bloser, Taylor P. Connor, Camden Ertley, Jason Legere, James M. Ryan, Sambid K. Wasti \\ Space Science Center, University of New Hampshire, Durham, NH 03824
}

\begin{abstract}
The Gamma RAy Polarimeter Experiment (GRAPE) was first flown on a 26-hour balloon flight in the fall of 2011. GRAPE consists of an array of Compton polarimeter modules (based on traditional scintillation technologies) designed to operate in the energy range from $50 \mathrm{keV}$ up to $500 \mathrm{keV}$. The ultimate goal is to operate GRAPE in a wide FoV configuration for the study of gamma-ray bursts. For the first (demonstration) balloon flight, GRAPE was configured in a collimated mode to facilitate observations of known point sources. The Crab nebula/pulsar, the active Sun, and Cygnus $\mathrm{X}-1$ were the primary targets for the first flight. Although the Crab was detected, the polarization sensitivity was worse than expected. This paper will review the plans for the next GRAPE balloon flight, which is scheduled to take place in the fall of 2014 from Ft. Sumner, NM. These plans involve several modifications designed to improve the polarization sensitivity, including an expansion of the array of polarimeter modules from 16 to 24 and improvements to the instrument shielding. Sensitivity estimates of the resulting instrument, based on GEANT4 simulations, will be presented.
\end{abstract}

Keywords: X-ray, gamma-ray, balloon, polarimeter, polarimetry

\section{INTRODUCTION}

The Gamma Ray Polarimeter Experiment (GRAPE) has been developed with the long-term goal of providing important observations of gamma-ray burst (GRB) and solar flare polarization in the 50-500 keV energy band. The engineering model (consisting of a single polarimeter module) flew on a small balloon payload in June of 2007. A balloon flight of the full GRAPE payload (consisting of 16 polarimeter modules) took place on September 23-24, 2011 from Ft. Sumner, NM. The primary goal of this first flight was to demonstrate the capability of the GRAPE design by measuring the polarization of the Crab Nebula. This was seen as a prelude to much longer duration balloon flights, with a modified payload configuration, that would allow us to measure polarization from a statistically significant sample of GRBs (and perhaps also solar flares).

\section{THE GRAPE POLARIMETER}

The physical process used to measure polarization in the 50-500 keV energy range is Compton scattering. In the plane perpendicular to the incident photon direction, Compton scattered photons tend to be scattered at right angles with respect to the incident electric field. In the case of an unpolarized beam of incident photons, there will be no preferred azimuthal scattering angle; the distribution of azimuthal scatter angles will therefore be uniform. In the polarized case, since there will be a preferred direction, the distribution of azimuthal scatter angles will be asymmetric (Fig. 1). For a given level polarization, the magnitude of the asymmetry is largest at low energies (below $100 \mathrm{keV}$ ) and is most pronounced for Compton scatter angles $(\theta)$ near $90^{\circ}$. The precise shape of this asymmetric distribution can be used to infer both the magnitude and direction of polarization. An accurate measure of this distribution requires determination of the scatter geometry and kinematics of individual photons.

The GRAPE design is modular. ${ }^{1-6}$ The detection concept of a single module is shown in Fig. 2. An array of opticallyisolated scintillator elements is placed on the front end of a single multi-anode photomultiplier tube (MAPMT), which is used to measure both the scatter geometry and the energy deposits associated with a Compton scattered photon. Two types of scintillator are used. Low-Z plastic scintillator elements (shown in grey) are used as the medium for Compton scattering the incident photons. High-Z inorganic scintillator crystals (calorimeter elements, shown in red) are used as a medium for maximizing the absorption probability of the scattered photon and for defining the azimuthal scatter angle. Each rectangular scintillator element is 5-mm across and 5-cm long. Although there is some flexibility in the geometric layout of the scintillator elements (the relative placement of plastic and calorimeter elements), the specific layout in Fig. 2 was chosen, based on extensive simulations with GEANT4, to maximize on-axis sensitivity.

${ }^{1}$ Mark.McConnell@unh.edu; phone +1-603-862-2047; fax +1-603-862-3584

UV, X-Ray, and Gamma-Ray Space Instrumentation for Astronomy XVIII, edited by

Oswald H. Siegmund, Proc. of SPIE Vol. 8859, 885909 - (c) 2013 SPIE

CCC code: $0277-786 \mathrm{X} / 13 / \$ 18 \cdot$ doi: $10.1117 / 12.2024145$

Proc. of SPIE Vol. 8859 885909-1 
Fine energy resolution was not a significant driver in the GRAPE design, as it is not of paramount importance in the study of GRBs and solar flares. The most important drivers were sensitivity and low cost. For the plastic scattering elements, the chosen material was EJ-204 (Eljen Technology). For the calorimeter elements, non-hygroscopic CsI(Tl) scintillator (acquired from Proteus, Inc.) was chosen for its ease of use, good spectral match to traditional bi-alkali photocathodes, good light output, and relatively low cost. The relatively small neutron interaction cross section in CsI also serves to reduce the background due to neutrons, a potentially significant background source for polarimeter designs consisting entirely of plastic scintillator. ${ }^{7}$

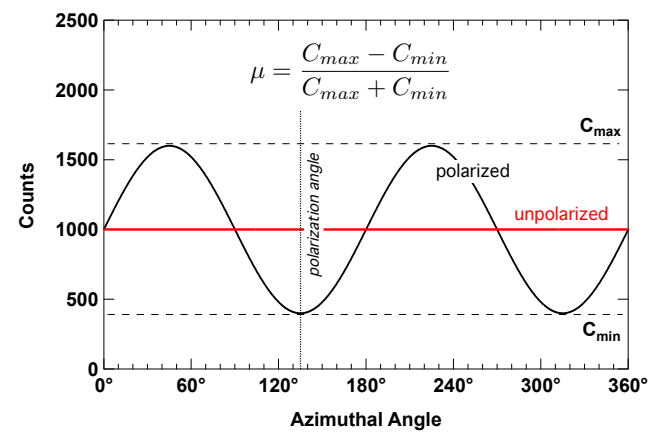

A single flat-panel MAPMT (Hamamatsu H8500) is used for Figure 1. The modulation pattern produced by simultaneous readout of both plastic and calorimeter elements. The Compton scattering of polarized radiation. The H8500 provides an $8 \times 8$ array of independent anodes, with $5 \mathrm{~mm}$ minimum of the modulation pattern defines the anodes arranged on a pitch of $6 \mathrm{~mm}$. Each scintillator element is co- plane of polarization of the incident flux. The aligned for readout by a single anode. The MAPMT itself measures 52 modulation factor $(\mu)$ is an instrument $\times 52 \mathrm{~mm}^{2}$ in area and $28 \mathrm{~mm}$ in depth, providing for a relatively parameter that quantifies the magnitude of the compact design. asymmetry.

An ideal polarimeter event involves the interaction of an incident photon in a plastic element followed by the absorption of the scattered photon in a calorimeter element. The locations of the two hit elements define the azimuthal scatter angle. These events (which we refer to as plastic-calorimeter or PC events) are identified as having triggered one (and only one) plastic element in coincidence with one (and only) calorimeter element. Other types of Compton scattering events are also possible. For example, one can have photons that scatter from one plastic element to another (PP events) or photons that scatter from one calorimeter to another (CC events). Although PC events dominate over all other event types, $\mathrm{CC}$ events become important contributors to the overall response of the polarimeter at energies above $200 \mathrm{keV}$. In an array of modules, scattering events between modules can also contribute.

Although the readout of the scintillator array by a single MAPMT has its advantages, it also brings with it a significant amount of optical crosstalk between adjacent elements. Although the scintillator elements are optically isolated from one another, crosstalk between adjacent anodes arises from the lateral spreading of the optical light as it exits the bottom end of a scintillator element. In an effort to ameliorate this optical crosstalk, circuitry has been developed to identified events that have been contaminated by crosstalk between adjacent scattering and calorimeter elements. The design takes advantage of the significantly different rise times of plastic and CsI scintillators by identifying a signal from a given anode that exhibits a timing signature characteristic of an adjacent scintillator element, rather than the scintillator associated with that particular anode. In this way, events associated with optical crosstalk between anodes with different type scintillator are prevented from getting into the data stream. However, events that do get into the data stream may still be subject to crosstalk between adjacent anodes with the same scintillator type. The analysis of data is therefore still complicated (although to a much lesser degree) by the presence of optical cross-talk between adjacent anodes within a module.

Various methods have been developed for handling remaining crosstalk-related issues. For example, crosstalk effects can be avoided to a great extent by looking only at those events whose interaction sites are in non-adjacent anodes, since crosstalk is typically limited to anodes that are directly adjacent to one another. In the case of events with adjacent anode signals, simulations of the optical light distribution are being used to delineate the intrinsic event signature from crosstalk effects.

Polarization measurements can be hampered by a variety of systematic effects that may influence the polarization measurement, leading to the degradation of an existing polarization signal or the generation of a false polarization signal. Systematic effects can arise, for example, from variations in detector efficiency, principle, detector thresholds, or the local background. In the GRAPE design, asymmetries a MAPMT. 

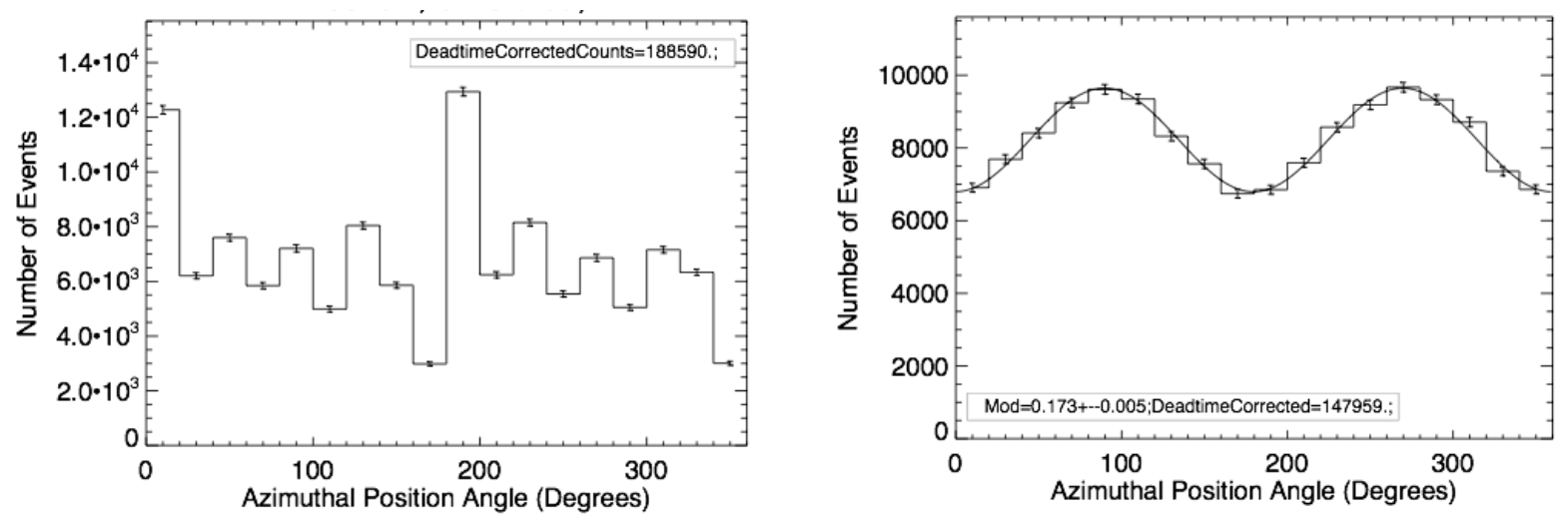

Figure 3. Calibration data showing scatter angle distributions for $288 \mathrm{keV}$ polarized photons. The left histogram is for an unrotated module. The right histogram is for a module continuously rotated about its pointing axis. Without the rotation, the polarization signal is completely lost.

result largely from the fact that only certain azimuthal scatter angles are allowed by the geometrical arrangement and discrete nature of the scintillator elements. Although these effects can be modeled by simulations or (in some cases) empirically measured, instrument rotation about the pointing direction is also known to be quite effective (as demonstrated in Fig. 3). However, rotation works well only for steady sources of radiation or sources that vary on timescales much longer than the instrument rotation period. In the case of transient sources with timescales less than a single rotation period (such as might be expected for solar flares or GRBs), rotation becomes far less effective, in which case alternative methods must be employed. Since the primary objective of the demonstration balloon flights has been to measure the Crab polarization, module rotation (with a period of 20 minutes) has been incorporated into the payload design.

\section{THE GRAPE BALLOON PAYLOAD}

For the 2011 balloon flight, the detector assembly consisted of 16 modules arranged in a $4 \times 4$ array. Each module (Fig. 4) had 36 optically isolated plastic elements surrounded by 28 optically isolated CsI(Tl) elements, all read out by a single MAPMT. Four 16-channel analog electronics boards processed the anode signals. Each of the 64 channels included a fast discriminator to generate triggers for the hit scintillator elements and a slow shaper with peak-detect-and-hold circuitry that formed part of the pulse height measurement. The module electronics also included an interface board and a PICbased logic board with fast coincidence circuitry to recognize coincident and anti-coincident events, validate each event, multiplex and digitize the pulse heights and assemble event messages for further processing.

Each individual module had its own cylindrical collimator, consisting of an $\mathrm{Al}$ tube $(7.6 \mathrm{~cm}$ OD with $1.2 \mathrm{~mm}$ thick walls) lined on the outside with $0.4 \mathrm{~mm}$ of $\mathrm{Pb}$ and extending $25 \mathrm{~cm}$ in front of the sensitive detector volume. This was designed to collimate the response of the detector array to within an acceptance angle of $\pm 10^{\circ}$.

The detector assembly (including the collimators) was completely enclosed on all sides by panels


Figure 4. Schematic of a polarimeter module (left) and a view of a fabricated flight module (with an aluminum cover over the scintillator array. 

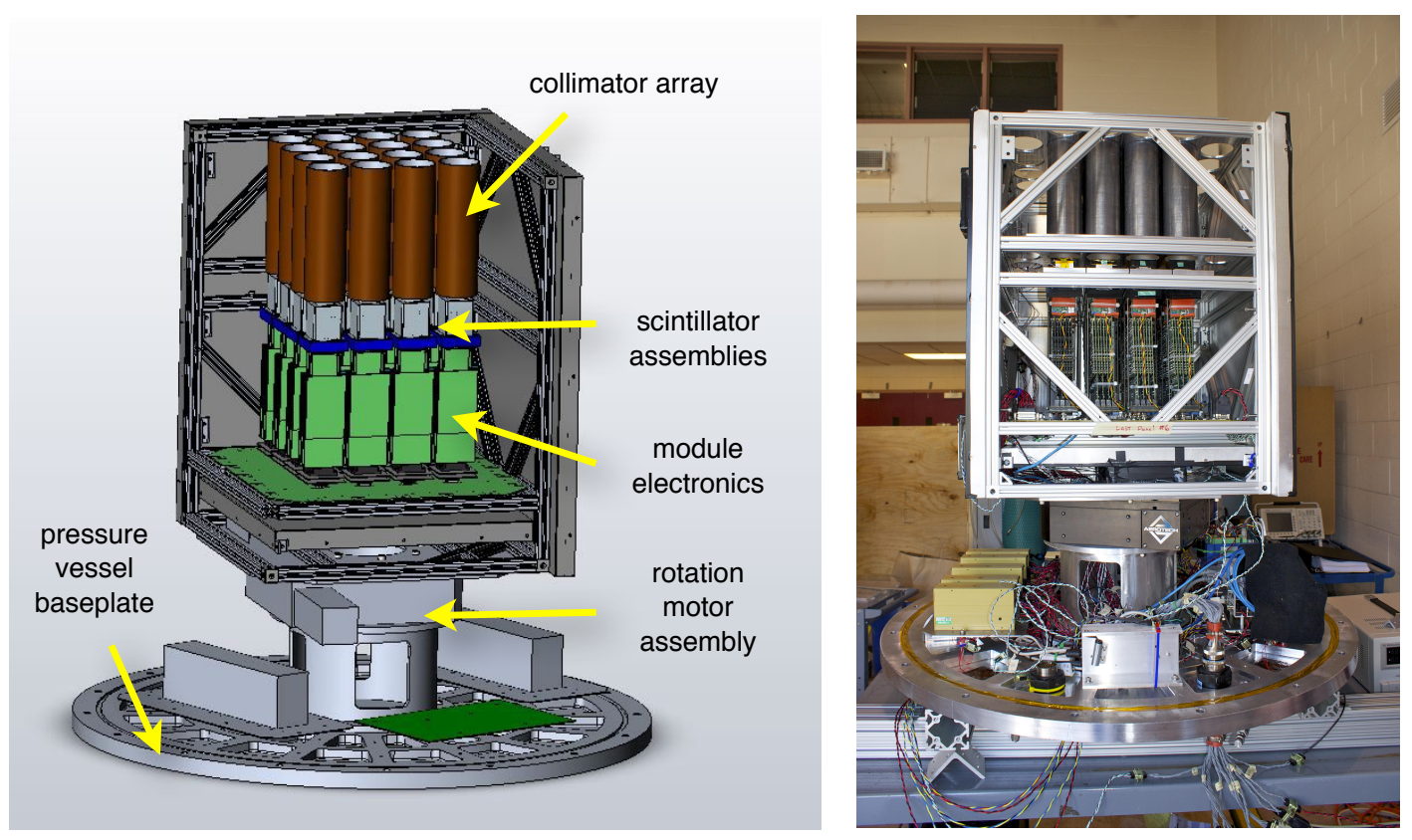

Figure 5. A schematic view of the instrument assembly (left) and a photo showing the instrument assembly in the lab (right, with side shielding removed).

composed of both passive and active shielding. Each panel had a 6-mm thick sheet of plastic scintillator which used a wavelength shifting (WLS) bar to collect the scintillation light and two PMTs, one on each end of the bar, to read out the signal. ${ }^{8}$ The multi-layer passive component of each shield panel consisted of $0.8 \mathrm{~mm}$ of $\mathrm{Pb}$ and $0.8 \mathrm{~mm}$ of Sn. The passive shields served to absorb atmospheric radiation from the sides, with the inner, lower-Z layer blocking fluorescent $\mathrm{X}$-rays from the outer, higher-Z layer. The top panel included cutouts in the passive shielding to allow for photons reaching each module.

The entire instrument (consisting of the detector assembly, collimators, and shields) sat on top of a motor assembly that rotated the instrument about the pointing axis (Fig. 5). The instrument was rotated through nearly $360^{\circ}$ once every 20 minutes. The table angle was changed in step sizes of $4^{\circ}$ with a dwell time at each step of $\sim 10$ secs. An encoder in the axial drive system measured and recorded the time-tagged orientation.

The instrument system electronics included a power supply and distribution module, a module interface board and an instrument computer. The detector modules plugged into the module interface board (MIB), which provided mechanical support for the detector array and served as the major system level interface between the detectors and the instrument computer. The instrument computer (which includes a TS-7200 processor) processed ground commands, packaged and buffered data, and coordinated the telemetry. Two flash disks were used for on-board data storage. The instrument power supply and distribution module converted and distributed the battery power.

All instrument components, along with the rotation table and associated electronics, were mounted inside an aluminum pressure vessel (Fig. 6), which was designed to maintain $1 \mathrm{~atm}$ of pressure throughout the flight. Instrument integration, test and debug activities could be conducted with the pressure vessel's sidewall / top dome structure removed to facilitate access. All instrument hardware was supported from the base plate, which also had ports for electrical and gas feed-throughs. The pressure vessel's top dome element was formed with 2-3 mm thick aluminum. An external frame supported and protected the pressure vessel and provided the mounting structure for the

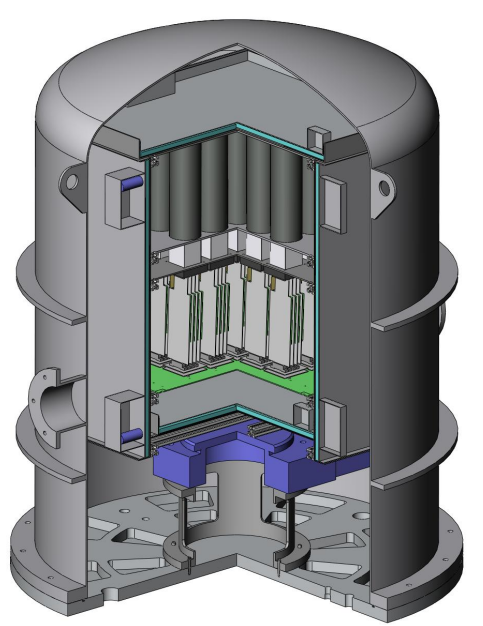

Figure 6. Cutaway drawing of the pressure vessel showing the interior placement of the instrument assembly. 
remaining payload components (Fig. 7).

The requirements of the attitude control system were driven by the need to maintain the source target within the $\pm 10^{\circ}$ FoV. Attitude control precision of $\pm 3^{\circ}$ and a pointing knowledge uncertainty of $\pm 0.5^{\circ}$ were deemed adequate. A NASA-supplied rotator (designed to provide solar panel orientation during LDB flights) provided the means to control the azimuthal orientation of the gondola. An elevation drive mechanism controlled the elevation angle of the pressure vessel and the enclosed instrument assembly. A commercial differential GPS unit (ADU 5) was used to provide the gondola's 3dimensional orientation at frequent intervals. The elevation angle of the pressure vessel pointing axis was measured with an inclinometer and recorded along with the time-tagged azimuth information.

Based on thermal modeling, coupled with the desire to maintain the instrument temperature between $-10^{\circ}$ and $+30^{\circ} \mathrm{C}$, several heating elements were installed at strategic locations within the pressure vessel. A total of 180 Watts, on average, was allocated for heaters.

In anticipation of future LDB flights, the CSBF mini-SIP flight system was employed for the telemetry interface, utilizing CSBF transmitters for line-of-sight (LOS) telemetry of the low- and high-rate science data and for commanding. All raw data was also stored on board using flash drives.

Each module was independently calibrated before instrument integration. An energy calibration was generated for each of the 64 anodes (both plastic and CsI) using calibration sources with photon energies ranging from $22 \mathrm{keV}$ to $356 \mathrm{keV}$. Total energy resolution varies from $\sim 50 \%$ at $60 \mathrm{keV}$ to $\sim 20 \%$ at $500 \mathrm{keV}$. For measuring the polarization response (c.f., Fig. 3), a beam of (electronically tagged) polarized photons was generated in the lab using calibration source photons scattered by $\sim 90^{\circ}$ in a block of plastic scintillator. Calibrations were also performed at the instrument level after payload integration, to insure stable module calibrations, to calibrate intermodule events, and to provide a baseline for detection rate asymmetries.

Processing of the event data starts with the conversion of pulse height to energy for each recorded anode signal. (Up to eight anode signals are recorded for each event.) Valid events are then identified based on event class (C, PC or CC events), the relative positions of the triggered anodes and evidence of crosstalk. A check on the Compton kinematics is then made to insure that the energy deposits are consistent with realistic Compton scatter angles. An azimuthal scatter angle is then calculated (in instrument coordinates) for each valid event. This angle is then projected onto the sky, taking into account the instrument rotation table angle and the instrument pointing direction (azimuth and elevation). The result is the position angle of the scatter vector (defined in the usual astronomical sense) with respect to the pointing direction. Histograms of position angle distribution can then be analyzed to search for evidence of polarization in the data, based on the known polarization response.

\section{THE 2011 BALLOON FLIGHT}

The first demonstration balloon flight of the full GRAPE payload took place on September 23-24, 2011 from Ft. Sumner, NM (Flight $624 \mathrm{~N})$. A total of 26 hours were spent at a float altitude that ranged from $3 \mathrm{gm} \mathrm{cm}^{-2}(130,000 \mathrm{ft})$ to more than $7 \mathrm{gm} \mathrm{cm}^{-2}(110,000 \mathrm{ft})$. The flight plan (Fig. 8) included pointed observations of the Crab and Cyg X-1. Based on results from INTEGRAL 9,10 it was expected that GRAPE would be able to measure the polarization from the Crab. On the other hand, the expected polarization level of Cyg X-1 is much lower, perhaps as low as 1 or $2 \%$, which would be below the polarization sensitivity for this configuration of GRAPE. Therefore, Cyg X-1 was expected to provide a good null test 
for polarization measurements. The flight plan also included the tracking of relatively "empty" fields through a full transit, designed to provide effective background measurements for the source observations. In addition, we planned to spend time looking at the Sun if there were signs of solar activity. As it turned out, the Sun was very active at the time of the flight.

\subsection{Crab Observations}

The analysis of the flight data was complicated in part by the balloon flight profile (Fig. 8) - in particular, the significant variations in altitude and its subsequent impact on the background rate. An additional complication resulted from the disruption of our flight plan (repointing of the instrument) due to solar activity. Although non-flare periods of solar observation provided additional background data, the change in flight plan resulted in the loss of a full empty-field transit observation. An added complication for the Crab observation itself was that sunrise took place during that time, inducing a rapid change in altitude that led to a rapid change in the instrumental background. (The payload transitioned from a relatively high background environment to a relatively low background environment near the middle of the Crab observation.) Background modeling therefore proved to be a challenge.

A Principle Component Analysis (PCA) was developed for analyzing the highly variable background. Several different segments of the flight during empty-field or weak-source observations were chosen as input to the PCA background model. (Data from Fermi-GBM showed Cyg X-1 to be in a very low state at these energies and below our sensitivity.) The background model could then be used to extract, for a selected energy and time interval of the source observation, the total number of source counts. An energy-loss spectrum for the source from individual time intervals or from the full observation could then be assembled and de-convolved to extract a source photon spectrum.

Since previous reports of polarization from the Crab have been derived from the un-pulsed regions of the pulsar pulse profile (i.e., the emission from the nebula), a detailed analysis of the Crab data required determining the pulsar phase for each recorded event and then incorporating the phase data into the analysis. Pulse profiles for the phase-tagged events were used to confirm proper pulsar phase determination by comparison with the known Crab phase profile at these energies.

The polarization measurement required that an analysis be performed (incorporating the PCA modeling) using the scatter angle histograms extracted from each time, energy and phase periods. Fig. 9 shows the result derived using data from the un-pulsed and inter-pulse regions of the Crab phase diagram (phases 0.5-0.88 and 0.14-0.25, respectively). Fitting the Crab data to the expected $\sin 2 \theta$ functional form for a polarization signal (Fig. 1) results in a derived polarization level of $66 \%( \pm 22 \%)$ and a polarization direction lying at a position angle of $154^{\circ}\left( \pm 21^{\circ}\right)$. These numbers are both consistent with the INTEGRAL measurements.

\subsection{Solar Flare Observations}

At the time of the flight, it had become apparent that there was a significant opportunity for a potential solar flare observation. Two active regions (1295 and 1302) were producing significant $\mathrm{M}$ - and X-class activity. An X1.4 flare from region 1302 took place on Sep 22, the day before the flight. Solar observations therefore took place both at the beginning of the flight and at the end of the flight. There was no significant flare activity during the first solar observation period. An X1.9 flare from region 1302 took place between solar observations, when the payload was in darkness! There

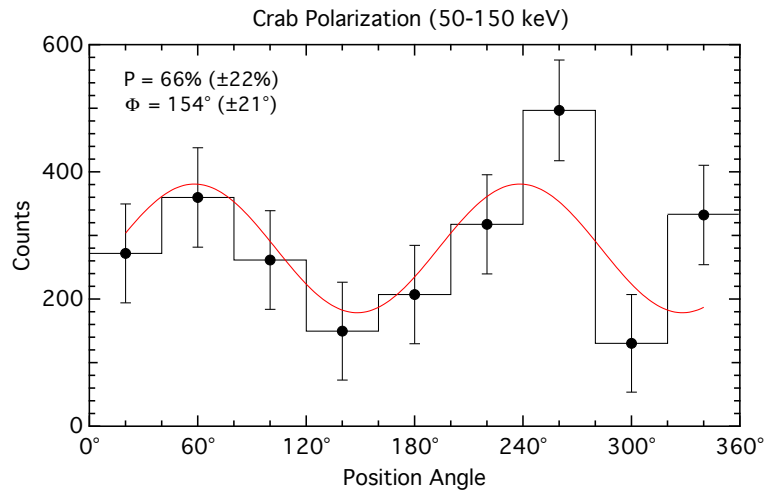

Figure 9. Preliminary polarization results for both the Crab nebula and a selected background (non-source) interval of data. 


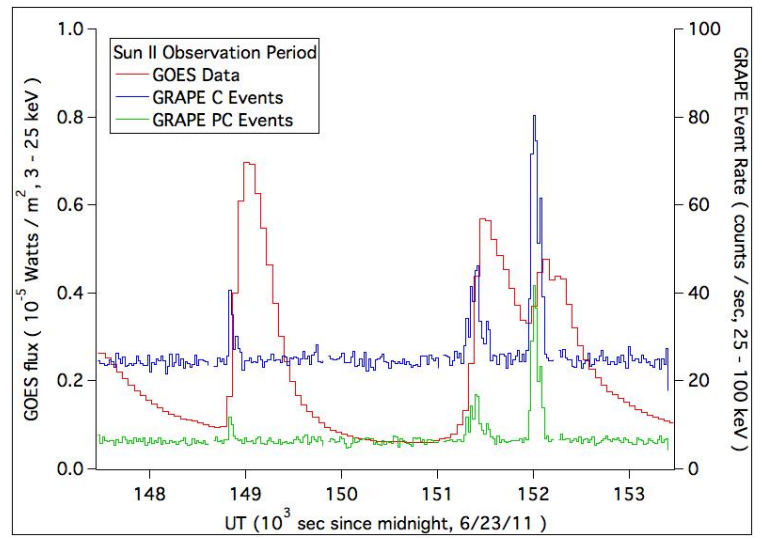

Figure 10. Time history of the solar flare events. The first peak represents an M3.1 flare. The second two peaks represent an M2.8 flare.

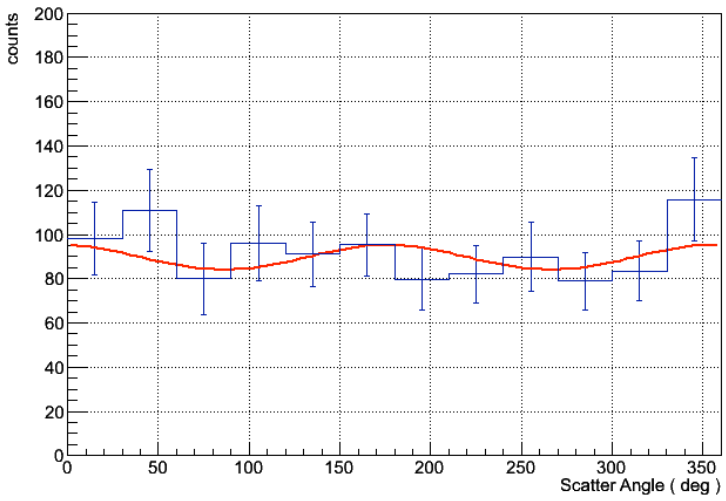

Figure 11. Polarization measurement for the last (and largest) peak shown in Figure 10. No clear evidence of polarization is evident.

were two smaller M-class flares that took place during the second solar observation period. GRAPE recorded a significant increase in counting rate for both events (Fig. 10).

The analysis of these data differed from that of the Crab in two important ways. First, the background estimate for the flare comes from an average of the background just before and after the flare. (The background was fairly stable during this part of the flight.) Second, the duration of each flare is much shorter than the 20 minute rotation period of the instrument, so instrument rotation could not be relied on to eliminate systematic effects in the data. Data from the rotation periods before and after that of the flare, collected over the same range of table angles, was used as a background. The scatter angle distribution derived from these "unpolarized" background data was used to correct the scatter angle distribution of the flare data for systematic effects. Fig. 11 shows the result from an analysis of the brightest peak in Fig. 10, which is associated with AR 1295. No clear evidence of a polarization signal can be seen, with an upper limit of $\sim 30 \% .^{11}$

\subsection{Flight Background}

Instrument background simulations take into account the detailed mass configuration of the payload and a variety of background sources. The mass model includes everything contained with the pressure vessel (modules assemblies, collimators, shielding, rotation platform, etc.) along with the pressure vessel itself. At these energies, the primary background components are the diffuse cosmic $\gamma$-ray flux entering the instrument aperture and the leakage of atmospheric $\gamma$-rays through the shields. The simulations also include primary and atmospheric cosmic rays (protons and electrons) as well as atmospheric neutrons. The parameterizations of the various components come from Gehrels ${ }^{12}$ for the $\gamma$ rays, Mizuno et al. ${ }^{13}$ for the charged particles, and Armstrong et al. ${ }^{14}$ for the atmospheric neutrons. The background modeling is appropriate for an atmospheric depth of $3.5 \mathrm{~g}$ $\mathrm{cm}^{-2}$ at the latitude of Ft. Sumner, NM. Figure 12 shows the contribution of the various background components (for PC events), along

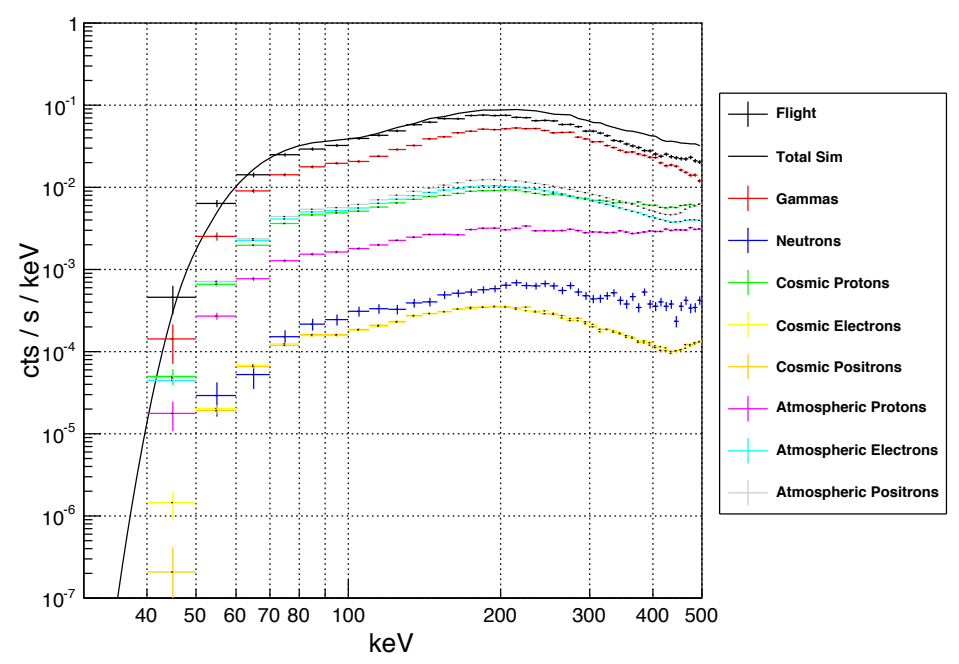

Figure 12. Simulated background spectrum for PC events, showing the various contributions to the background. The corresponding spectrum from flight data is shown for comparison. 
with a comparison between the simulated background and the flight background. The flight data agrees well within simulations at lower energies, but at higher energies, the flight background is lower than predicted by simulations. This discrepancy may result from events being discarded in the processing due to crosstalk effects. Simulations of each separate event class (PC, CC and C events) are currently being used to help refine the processing of the flight data. These simulations have also been used to optimize the payload configuration for the 2014 balloon flight.

\section{PLANS FOR THE 2014 BALLOON FLIGHT}

The 2011 balloon flight was designed to demonstrate the ability of GRAPE to conduct scientific measurements. The collimated configuration was optimized for observations of steady point sources of radiation. The goal was to demonstrate the capabilities of GRAPE by making a positive polarization measurement of a source of radiation (the $\mathrm{Crab}$ ) that is thought to be polarized at these energies. Although the preliminary results (Fig. 9) suggest a marginally positive measurement, the polarization sensitivity of GRAPE appears to have been worse than expected based on pre-flight predictions. The data indicate both a lower source rate (roughly a factor of two lower) and a higher background rate (almost twice as high) than had been estimated.

The lower source rate results primarily from the larger atmospheric depth throughout much of the flight (almost $7 \mathrm{gm} \mathrm{cm}^{-2}$ as opposed to the planned $3.5 \mathrm{gm} \mathrm{cm}^{-2}$ ). The higher background rate can also be attributed, in part, to the larger atmospheric depth. However, a major factor in the higher background rate was the thickness of the

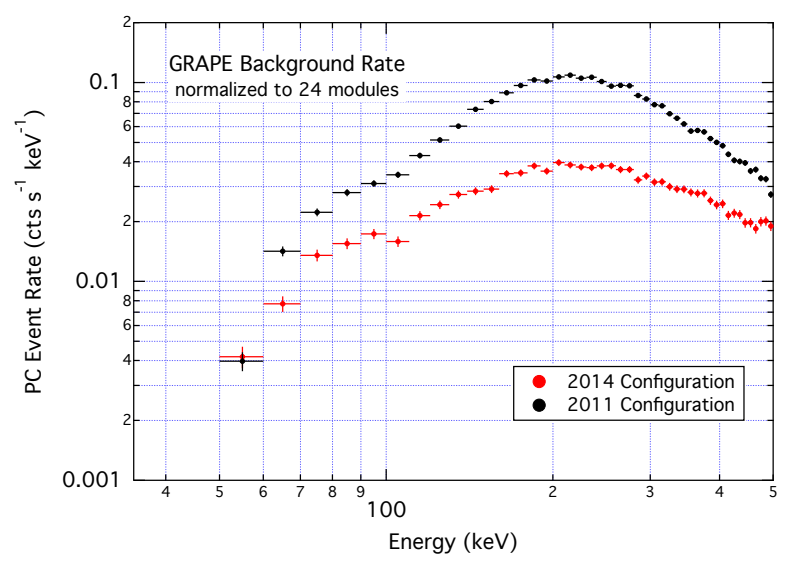

Figure 13. Simulated background (PC events) for both the 2011 and 2014 configurations of GRAPE. The 2011 background has been normalized from 16 to 24 modules. passive shielding. Pre-flight background estimates had been based on data from a 2007 engineering model balloon flight, which had employed considerably thicker passive shielding. The 2007 flight employed passive shielding of $4 \mathrm{~mm} / 1$ $\mathrm{mm} / 1 \mathrm{~mm}$ of $\mathrm{Pb} / \mathrm{Sn} / \mathrm{Cu}$. The $2011 \mathrm{flight}$ employed passive shielding of $0.8 \mathrm{~mm} / 0.08 \mathrm{~mm}$ of Pb / Sn. Thinner shielding was employed in 2011 to reduce the torque on the rotation motor at large zenith angles, but this clearly came at the cost of a much higher background.

Preparations are currently underway for a second demonstration flight in the fall of 2014. The configuration for the 2014 flight will be essentially the same as that for the $2011 \mathrm{flight}$, but it will incorporate several design changes to maximize the polarization sensitivity. The most important of these changes include:

- Thicker passive shields. Increasing the Pb thickness from $0.8 \mathrm{~mm}$ to $4.2 \mathrm{~mm}$ will not only reduce the background, but will also extend the effective energy range to higher energies (up to at least $300 \mathrm{keV}$ ).

- Expanded array size. The hardware has been designed to accommodate up to 32 polarimeter modules, twice as many as in the first flight. The number of polarimeter modules will be increased from 16 to 24 for the 2014 flight, still below the capacity of the payload, but sufficient to provide a significant increase in sensitivity.

The sensitivity of a polarimeter is defined in terms of the Minimum Detectable Polarization (MDP), which refers to the minimum level of of polarization detectable with a given observation (or, equivalently, the apparent polarization arising from statistical fluctuations in unpolarized data). The value of the MDP depends on the source parameters (fluence, spectrum, etc.), energy-dependent polarimeter characteristics, and energy-dependent background. At the $99 \%$ confidence level, the MDP is defined by, ${ }^{15}$

$$
\operatorname{MDP}(E)=\frac{4.29}{\mu_{100}(E) F_{S}(E) A_{e f f}(E)} \sqrt{\frac{F_{S}(E) A_{e f f}(E)+R_{B}(E)}{T_{o b s}}}
$$

where $\mu_{100}$ is the modulation factor (c.f., Fig. 1) for a $100 \%$ polarized source, Fs is the source flux (photons $\mathrm{cm}^{-2} \mathrm{~s}^{-1}$ ), $\mathrm{A}_{\text {eff }}$ is the instrument effective area $\left(\mathrm{cm}^{2}\right), \mathrm{R}_{\mathrm{B}}$ is the background count rate $\left(\mathrm{cts} \mathrm{s}^{-1}\right)$, and $\mathrm{T}_{\mathrm{obs}}$ is the observing time (s). 


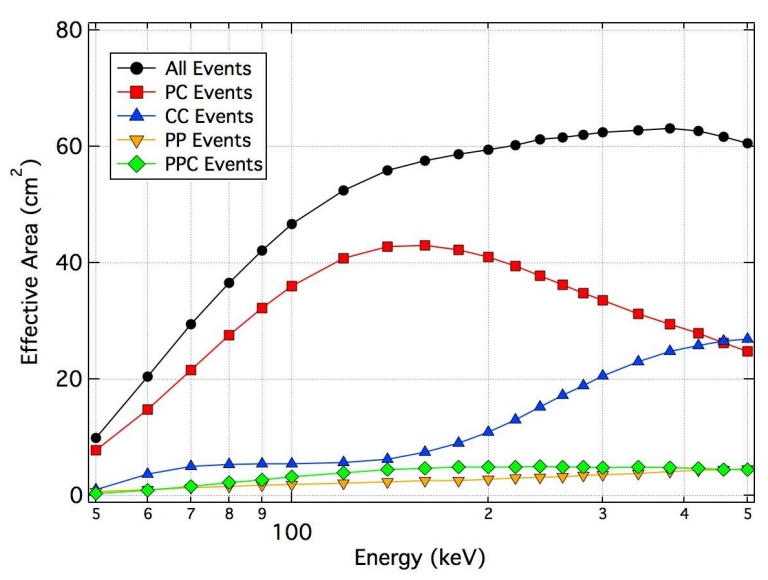

Figure 14. Simulated effective area of the full payload for various event types $(\mathrm{P}=$ plastic and $\mathrm{C}=$ calorimeter $)$.

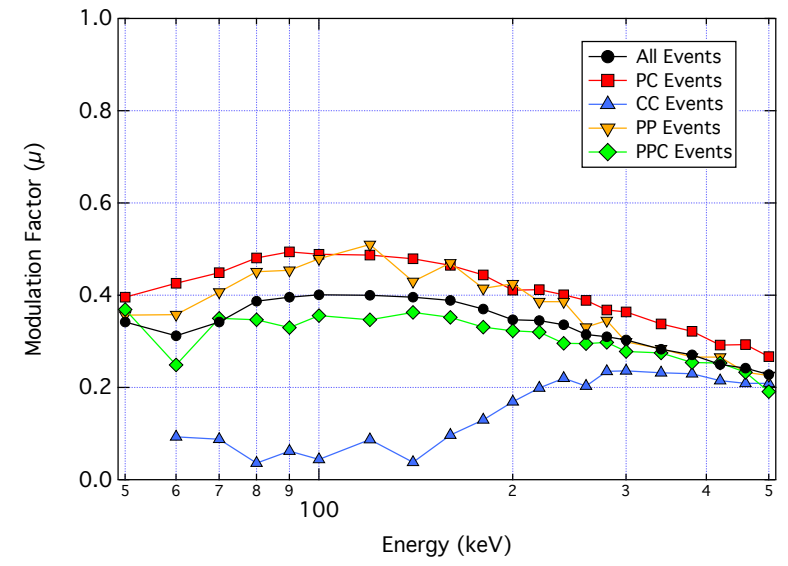

Figure 15. Simulated modulation factor of the full payload for various event types $(\mathrm{P}=$ plastic and $\mathrm{C}=$ calorimeter $)$.

GEANT4 simulations have been used to evaluate the effectiveness of the increased passive shielding. The results are shown in Fig. 13, which shows the predicted PC event background rate for the 2014 configuration, along with equivalent results for the 2011 configuration (scaled from 16 to 24 modules). The simulations predict a (per module) background that will be 2-3 times lower in the 2014 instrument configuration.

The instrument response parameters as a function of energy have also been studied using GEANT4 simulations. The simulations considered several different event classes, including PC, PP, CC, and PPC events. CC events between separate modules were also included.

The effective area and modulation factor are shown as a function of energy in Figs. 14 and 15, respectively. The response is dominated by PC events at low energies, but CC events also become quite important at energies above 250 $\mathrm{keV}$. The hardware triggering system requires a signal from a calorimeter (C) element and the pulse-shape circuitry rejects events with crosstalk between calorimeter and plastic events. Crosstalk in PC and CC events is handled effectively by this system. The system is also moderately effective at handling crosstalk associated with PPC events. In most PPC events, the azimuthal scatter angle can be derived assuming that the photon scatters first between the two plastic elements before interacting in the calorimeter. The hardware has not been designed to handle PP events, but this is of little consequence, since the simulations show that PP events contribute very little to the effective area.

The flight plan in 2014 will follow that of the 2011 flight, concentrating on observations of the Crab, Cyg X-1 and perhaps the Sun. We will impose more stringent altitude requirements, with the goal of maintaining an altitude in excess of 120,000 feet for the duration of the flight. This will keep the background rate relatively low (and stable) and the source rate high. For an atmospheric depth of $3.5 \mathrm{gm} \mathrm{cm}^{-2}$ and an exposure of 6 hours at an average zenith angle of $20^{\circ}$, simulations show that the 2014 configuration will provide an $50-150 \mathrm{keV}$ MDP (99\% CL) of $\sim 30 \%$. This is well below the $46 \%$ polarization level measured by INTEGRAL in the 0.1-10 MeV energy range. A successful demonstration flight in 2014 could lead to the first LDB flight of GRAPE as early as December of 2015, with the goal of accumulating GRB polarization measurements.

\section{REFERENCES}

[1] McConnell, M. L., et al., "Dedicated polarimeter design for hard x-ray and soft gamma-ray astronomy," Proc. SPIE 5165, 334-345 (2004).

[2] Legere, J., et al., "Developing a Compton polarimeter to measure polarization of hard x-rays in the 50-300 keV energy range," Proc. SPIE 5898, 413-422 (2005).

[3] McConnell, M. L., et al., "A hard X-ray polarimeter designed for transient astrophysical sources," IEEE Nuclear Science Symposium Conference Record, 1, $462-466$ (2005). 
[4] Bloser, P. F., et al., "GRAPE - A Balloon-Borne Gamma-Ray Polarimeter Experiment," ChJAA 6, 393-397 (2006).

[5] McConnell, M. L., et al., "GRAPE: a balloon-borne gamma-ray polarimeter," Proc. SPIE 7435, 74350J (2009).

[6] Connor, T. P., et al. (2006). "Plans for the first balloon flight of the gamma-ray polarimeter experiment (GRAPE)," Proc. SPIE 7732, 77324E (2010).

[7] Produit, N., et al., "POLAR, a compact detector for gamma-ray bursts photon polarization measurements." Nucl. Instr. Meth. 550, 616-625 (2005).

[8] Pendleton, G. N., et al., "FiberGLAST: a scintillating fiber approach to the GLAST mission." Proc. SPIE 3765, 12-21 (1999).

[9] Dean, A.J., et al., "Polarized Gamma-Ray Emission from the Crab." Science 321, 1183-1185 (2008).

[10] Forot, M., Laurent, P., Grenier, I. A., Gouiffès, C., \& Lebrun, F., "Polarization of the Crab Pulsar and Nebula as Observed by the INTEGRAL/IBIS Telescope." Astrophys J 688, L29-L32 (2008).

[11] Ertley, C., et al. (2013), in preparation.

[12] Gehrels, N., "Instrumental background in balloon-borne gamma-ray spectrometers and techniques for its reduction." Nucl. Instr. Meth. 239, 324-349 (1985).

[13] Mizuno, T., Kamae, T., Godfrey, G., et al., "Cosmic-Ray Background Flux Model Based on a Gamma-Ray Large Area Space Telescope Balloon Flight Engineering Model.” Astrophys J 614, 1113-1123 (2004).

[14] Armstrong, T. W., et al., "Calculations of neutron flux spectra induced in the earth's atmosphere by galactic cosmic rays." J. Geophys. Res. 78, 2715-2726 (1973).

[15] Weisskopf, M. C., Elsner, R. F., O'Dell, S. L., "On understanding the figures of merit for detection and measurement of x-ray polarization.” Proc. SPIE 7732, 77320E (2010). 